\title{
A pilot investigation into brain-computer interface use during a lucid dream
}

\author{
Remington Mallett \\ Department of Psychology, University of Missouri at St. Louis \\ Department of Psychology, University of Texas at Austin
}

During lucid moments of a dream, the sleeper is aware of the dream as it is occurring, and as a result can often perform predetermined actions within the dream. This provides a unique opportunity for dream research, as lucid dreamers can send real-time signals from sleep to the external world. Historically, such sleep-to-wake communication from a lucid dream is executed via left-right eye movements, which places hard limitations on information transfer. Recent advances in biomedical equipment - specifically braincomputer interfaces - have resulted in headsets that use neural recordings to translate mental imagery into computer commands. In light of evidence suggesting that dreamed and imagined actions recruit similar neural resources, I considered the possibility that the same mental commands that are collected and translated from waking imagery could be similarly performed and detected from within a lucid dream. In this exploratory study with proof-of-concept intent, three participants were asked to use an Emotiv EPOC+ headset and companion software to map a mental motor command (pushing a block) with a resulting computer action (graphic of block moving forward). After waking training, participants attempted to induce a lucid dream while wearing the headset, and upon lucidity perform the same mental command. In two participants, subjectively reported lucid dream task completion was corroborated with video footage of the resulting computer graphic. These preliminary results suggest that a wake-trained brain-computer interface can be controlled from sleep and offer important directions for future dream communication and research.

Supplementary Information (SI) available at https://osf.io/7baw4/.

\section{Resource availability statement}

All data, code, and prior disseminated versions of this work are available at the OSF project site https://osf.io/mr7hf/.

\section{Acknowledgments}

I would like to thank Suzanne Welcome for essential mentorship and project guidance, Michelle Carr for comments on the manuscript, and lucid dreamers for their participation and insightful discussion. 


\section{Funding sources}

This work was funded by a Psi Chi Undergraduate Research Grant.

Conflict of interest statement

No potential conflicts of interest are declared.

Word count

Abstract - 240

Body - 3560 


\section{Introduction}

During a lucid dream, the (lucid) dreamer is aware of the dream state as it is occurring (Baird, Mota-Rolim, \& Dresler, 2019; LaBerge, Nagel, Dement, \& Zarcone, 1981). This awareness of the dream state often coincides with some level of volitional control over dream content and actions (Dresler et al., 2014; Stumbrys, Erlacher, Johnson, \& Schredl, 2014). The ability of a participant to perform specific actions within the dream led to the initial objective verification of lucid dreaming (Hearne, 1978; LaBerge et al., 1981). Eye movements and fist clenches within the dream have predictable and measurable external physiological correlates, which were used to send predetermined signals from a sleeping participant to the experimenter (detected via polysomnography). This method of communicating from a dream with left-right-left-right (LRLR) eye signals has become the de-facto standard for timestamping the moment of lucidity during relevant experiments (Baird et al., 2019) and has also been used as a method of communicating to the researcher in real-time from sleep (Appel, 2013; Fenwick et al., 1984; Konkoly \& Paller, 2019; Schatzman, Worsley, \& Fenwick, 1988).

However, this method of communication has limitations. Sending signals with eye movements or muscle clenches places a hard limitation on the amount of information that can be sent over a limited time. Participants with knowledge of Morse code have communicated from sleep by using left/right muscle clenches or eye movements as long/short tones to represent letters (Appel, 2013; LaBerge et al., 1981). While allowing for more complex signaling, this method's shortcoming is the length of time required to send a message. The duration of lucidity is limited, and thus Morse code messages from a lucid dream are typically restricted to just a few letters. A survey study found that participants reported an average estimated duration of about 14 minutes for their home lucid dreams (Stumbrys et al., 2014), although this might not be the same for laboratory lucid dreams. Further, Morse code is not a common language and requires significant effort for the participant to learn. More advanced eye-tracking methods have found that specific shapes can be drawn with orchestrated eye movement patterns (LaBerge, Baird, \& Zimbardo, 2018), but it is unclear if this can serve as an effective real-time communication system (although see Appel, 2019). Thus, current sleep-to-wake signaling methods are largely limited to binary messages or numbers (e.g., $x$ amount of eye movements). The limitations of these signaling methods hinder research and the development of applications that utilize sleep-to-wake communication (Appel, Pipa, \& Dresler, 2018). Here, I propose a proof-of-concept for a communication method that could overcome these current limitations.

Current sleep-to-wake communication methods are effective in-so-far-as (lucidly) dreamed actions share a similar physiology as waking actions and imagery (Dresler et al., 2011; Erlacher \& Schredl, 2008). The notion that imagined actions recruit similar neural resources as waking is also a key premise behind brain-computer interface $(\mathrm{BCl})$ 
technology (Chaudhary, Birbaumer, \& Ramos-Murguialday, 2016; Lebedev \& Nicolelis, 2006). BCls recording neural signals from motor cortex have allowed clinically immobilized patients to control robotic arms or other digital computer signals using only mental imagery or movement intentions (Wolpaw, Birbaumer, McFarland, Pfurtscheller, \& Vaughan, 2002). Most lucid dreams occur in rapid eye movement sleep (LaBerge, 1988; LaBerge, Levitan, \& Dement, 1986), at which stage muscle atonia increases motor neuron inhibition, effectively paralyzing the sleeper with the exception of eye movements and muscle twitches (Chase \& Morales, 1990). Therefore, a clinically immobilized patient and an actively lucid dreaming participant might be in a similar situation of being both conscious of their waking body and fully intent on moving their limbs, despite being unable to do so. Accordingly, I hypothesized that due to the ability of a lucid dreamer to perform predetermined actions during a dream and the neural overlap between waking and lucidly dreamed actions, a $\mathrm{BCl}$ that could be successfully controlled during waking could be similarly controlled during a lucid dream.

Experienced lucid dreaming participants were recruited to test this hypothesis in an exploratory pilot study. For each participant, a consumer-grade $\mathrm{BCl}$ (Emotiv EPOC+) was trained to distinguish between the presence and absence of a specific mental imagery task, and then upon lucidity the same mental task was performed. All participants subjectively reported performing the task during a lucid dream, as indicated by written dream reports and lucidity-focused questionnaires. When participants were asleep but not performing the task, the $\mathrm{BCl}$ showed little detection of the task. Importantly, when participants performed the task during a lucid dream (prefaced with LRLR eye movements), the $\mathrm{BCl}$ showed increased task detection levels.

Due to low-quality equipment and lack of access to the BCl's raw data, this preliminary investigation warrants scrutiny and further verification. Yet, the results suggest that a $\mathrm{BCl}$ trained on a waking mental imagery task can then be controlled during sleep by performing the same task when volition reappears in lucid dreaming. While the current study focused on $\mathrm{BCl}$ detection of a single command, the rapid development and potential of $\mathrm{BCl}$ research suggest that $\mathrm{BCl}$ technology could offer a solution to existing sleep-to-wake communication limitations.

\section{Methods}

Participants

Three experienced lucid dreamers (reporting successful lucid dream induction in $\geq 25 \%$ of attempts) participated in the current pilot study. All participants were recruited via personal contact except sub-001 (the author). All participants were male between the ages of 25 and 35 years (sub-001=26, sub-003=25, sub-004=35). One volunteer (sub002) dropped out after consent and did not follow through with any steps of participation. 
Participant sub-001 reported completing the task, but due to a computer malfunction (no data was recorded during the sleep session) is not included in analyses (but dream report and questionnaires are included in SI). Participant sub-003 was a narcoleptic.

82 Narcoleptics show an increased tendency towards lucidity during dreams (Dodet, 83 Chavez, Leu-Semenescu, Golmard, \& Arnulf, 2015; Rak, Beitinger, Steiger, Schredl, \& 84 Dresler, 2015) and are increasingly used in lucid dreaming research (e.g., Oudiette et al., 2018). Research was performed in accordance with the University of Missouri Institutional Review Board.

\section{Brain-computer interface}

The $\mathrm{BCl}$ setup was an Emotiv EPOC+ headset (Figure 1A) and companion software (Xavier Controlpanel 3.0.0.44; Xavier TestBench 3.0.0.37) running on a Macbook Pro laptop. The Emotiv EPOC+ is a 14-channel mobile electroencephalograph (EEG) system designed to maximize efficacy, mobility, and affordability (Figure 1A) while maintaining a level of reliability near laboratory-grade EEG systems (Badcock et al., 2013; Bobrov et al., 2011; De Vos, Kroesen, Emkes, \& Debener, 2014; Debener, Minow, Emkes, Gandras, \& Vos, 2012; Taylor \& Schmidt, 2012; Zich, De Vos, Kranczioch, \& Debener, 2015). The EPOC+ responds to $0.16-43 \mathrm{~Hz}$ using 14 (plus 2 reference) salinesoaked sensors at the following electrode locations: AF3, F7, F3, FC5, T7, P7, O1, O2, P8, T8, FC6, F4, F8, AF4 (references at left/right mastoids). The main feature of the Emotiv EPOC+ is the ability to build an EEG classifier that detects the intention of moving a virtual block and displays a corresponding graphic upon intention detection. The general layout for training and testing the EPOC+ is 1) a virtual block is displayed in the middle of the computer screen (Figure 1B), 2) a resting state, or non-task, EEG signal is collected under a neutral label, 3) EEG data 105 is collected under a push label while the 106 user performs a mental block-pushing task 107 (MBPT), 4) after steps 2 and 3 are 108 repeated, the user only has to perform the 109 MBPT and the virtual block will move 110 forward (i.e., push) in response. One 111 potential challenge in the current design 112 was that the back of the EPOC+ headset 113 protrudes a few inches, possibly making it 114 difficult to wear while sleeping. However, 115 slight modifications such as the addition of 116 neck support enabled the EPOC+ to be

A)

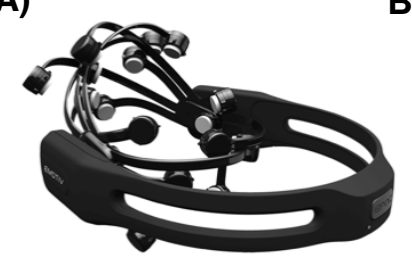

Figure 1. $\mathrm{BCl}$ setup. A) Emotiv EPOC+ headset. Participants were able to sleep with the headset on by lying on their backs and without support under the upper half of the head. B) Emotiv Controlpanel software. The mental blockpushing task (MBPT) involves imagining this virtual block moving forward (i.e., deeper) into the screen. During training, the block animates moving forward while the participant performs the mental task. During testing, the block only animates when the headset detects the MBPT. 
117 used during sleep. Battery life of the EPOC+ is reported as greater than 6 hours using

118 wireless connectivity (Bluetooth), which was ample time for the current study design.

\section{Lucid dream induction technique}

The study was designed such that participants could employ their preferred lucid dream induction technique (Price \& Cohen, 1988; Stumbrys, Erlacher, Schädlich, \& Schredl, 2012). Accordingly, the EPOC+ was applied either preceding an afternoon nap (sub-003) or during the morning/awake period of a wake-back-to-bed (WBTB; sub-001 and sub-004). WBTB is a common lucid dream induction technique that involves waking up after about five hours of sleep, staying awake for 10-90 minutes, and returning to bed with the intention of having a lucid dream (LaBerge, 1980; Stumbrys et al., 2012). The potential lucid dream generally occurs anywhere from sleep onset to one hour into the second sleep period.

\section{General procedure}

Participants completed a training phase of approximately 45 minutes during wake where they learned to push a virtual block with mental effort, followed by a test phase where they tried to induce a lucid dream and push the same virtual block during sleep. The goal of the training phase was to train the $\mathrm{BCl}$ on the distinction between a participant performing the MBPT and rest. The goal of the testing phase was to establish whether the same MBPT could be performed and detected during a lucid dream. Participants were allowed to complete a train-test sequence on multiple occasions. Because participants varied in their specific protocols as a result of different lucid dream induction techniques, the details of their procedures varied and are included in the Results section.

\section{Training phase}

After the $\mathrm{BCl}$ was properly set up on the participant, the $\mathrm{BCl}$ was trained on the MBPT. The participant was instructed to alternate performing the MBPT and a resting task for 8-second segments while EEG was recorded and mapped to Emotiv software labels push and neutral, respectively. For the MBPT, the participant was told: "Imagine pushing the block - however you choose to imagine it, all that matters is that the thought is consistent." For the resting task, the participant was told: "Clear your head." These instructions were a subtly modified version of those provided by Emotiv. Participants alternated between MBPT and rest mappings until they passed a qualitative evaluation of successful training. To evaluate $\mathrm{BCl}$ training, the participant again alternated between the MBPT and rest, but here the $\mathrm{BCl}$ only measured EEG and decoded the participant's thought process (MBPT vs. rest). If perfectly trained, the block graphic would move forward only and always during the MBPT, indicating 100\% decoding accuracy. This 
155 verification step was completed intermittently throughout the training phase until the $\mathrm{BCl}$ could accurately detect the MBPT on $~ 70 \%$ of attempts.

\section{Testing phase}

The testing phase consisted of the participant falling asleep with the $\mathrm{BCl}$ on, attempting to induce a lucid dream, and upon lucidity repeating the same MBPT they performed during training. As is customary in lucid dreaming studies, each participant was asked to first confirm the lucid dream state with a LRLR eye signal (Figure 2) before attempting the experimental task (Baird et al., 2019). This signal also serves to timestamp the beginning of dream task execution. Each participant was asked to repeat a sequence of the LRLR signal and 8-10 seconds of the MBPT as many times as possible once lucid in the dream. Computer activity was recorded and reviewed offline. Immediately upon awakening, participants were asked to complete a custom waking survey 169 that included a written dream report, specific questions about the MBPT 171 and LRLR signaling, the Dream 172 Lucidity Questionnaire (DLQ; 173 Stumbrys, Erlacher, \& Schredl, 174 2013) and the Lucidity and 175 Consciousness in Dreams scale

176 (LuCiD; Voss, Schermelleh-Engel, 177 Windt, Frenzel, \& Hobson, 2013).

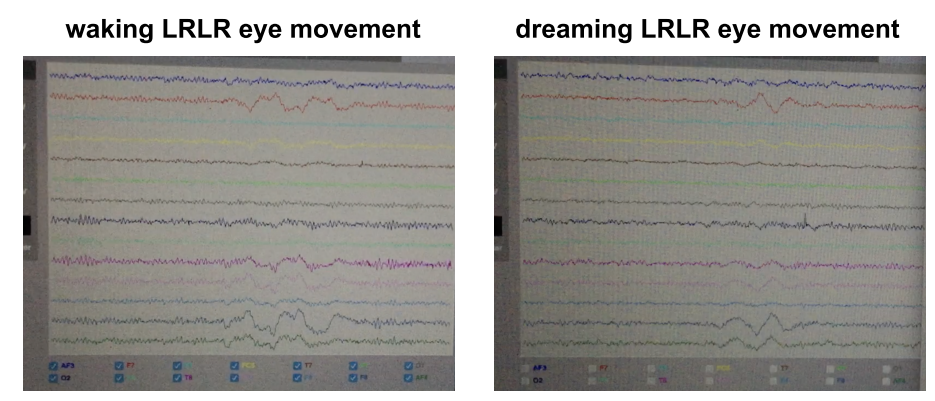

Figure 2. LRLR eye movements. Screenshots of what LRLR eye movements look like when performed during waking (left) and during a lucid dream (right). These are from participant sub-003, see Figure S1 for sub-004.

Analysis

Emotiv does not offer access to their machine learning algorithms or classifier decision output. To quantify the BCl's detection of the MBPT during sleep, the testing phase video was separated into 8-second segments centered around the first LRLR eye signal, and within each segment the "MBPT detection" score is the proportion of time that the display showed MBPT detection. To evaluate the ability of the $\mathrm{BCl}$ to detect the MBPT performed during a lucid dream, the MBPT detection scores during reported lucid dream MBPT completion were compared against all other MBPT detection scores (i.e., those from segments during sleep that the participant did not report performing the MBPT). Instructions to the participants were to attempt the MBPT during lucidity for 8-10 seconds after LRLR signaling, but to account for possible time discrepancies between lucid dreaming and waking (Erlacher, Schädlich, Stumbrys, \& Schredl, 2014), MBPT detection scores of the two segments following LRLR signaling were averaged, resulting in a single MBPT detection score to be compared against all others. The $p$-values were calculated as the proportion of null distribution MBPT detection scores that were greater than the 
194 single MBPT detection score from the lucid dream MBPT completion. Each participant's

195 empirical null distribution was bootstrapped by resampling with replacement 10000 times 196 from all MBPT detection scores outside of the lucid dream MBPT completion. This 197 analysis was performed individually on each test phase where the participant reported 198 completing the MBPT during a lucid dream. Analyses were performed using NumPy 199 (Oliphant, 2006) and Pandas (McKinney, 2010) in an IPython (Perez \& Granger, 2007) 200 environment. Data visualizations were performed using Matplotlib (Hunter, 2007).

\section{Results}

After waking training, the $\mathrm{BCl}$ was able to reliably detect the MBPT within each participant. In a following sleep session, participants were asked to become lucid while dreaming and then perform the same MBPT after a LRLR eye signal. To evaluate whether the $\mathrm{BCl}$ detected this task during sleep, MBPT detection scores from the time of lucid dream task completion were compared against the MBPT scores of all other sleep segments. Two participants successfully induced a lucid dream and self-reported performing the MBPT task after LRLR eye signaling while video was being recorded. The results from each of these two cases are reported separately below.

Participant sub-003 reported frequently becoming lucid during afternoon naps, and so performed the experiment during two afternoon sessions. During the first session sub003 reported becoming lucid but not performing the MBPT during the dream, and so returned for a second session. Participant sub-003 completed the training phase in a simulated sleep position in the hopes to mimic the test phase as much as possible. This consisted of lying down in the expected sleeping position (on back) with eyes closed and lights off. On the second train-test sequence, the training phase was stopped after approximately 30 minutes because the $\mathrm{BCl}$ was well-trained, as indicated by the small $95 \%$ confidence interval of the null distribution ([0,.52]; Figure $\mathbf{3 C}$; see also verification video clip*). Because all MBPT detection scores in the null distribution come from segments during sleep where the MBPT was not being performed, the spread of this distribution is a measure of how well the $\mathrm{BCl}$ was trained (where higher spread implies poorer training). Training was followed immediately by a nap and lucid dream MBPT attempt. During this nap, sub-003 reported successfully becoming lucid and completing the MBPT during the lucid dream (Figure 3A). DLQ scores indicate clear subjective

227 lucidity (Figure 3B). Participant sub-003 reported attempting the task twice consecutively 228 upon lucidity, and the averaged MBPT score of the two segments following LRLR

"https://osf.io/2gctz/ 
229 signaling was far outside the null distribution of non-task relevant MBPT test scores (.91, $230 \quad p<.0001$; Figure 3C; see video clip of MBPT during lucid dream ${ }^{\dagger}$.

\section{sub-003}

A)

... I was lucid ... then I remembered that I was in the lab at UMSL ... it triggered my memory to do the signal ... I just stood on the sidewalk, looked forward, and did the LRLR signal, then pushed for 8 seconds ... I was excited that I completed the task...

B)

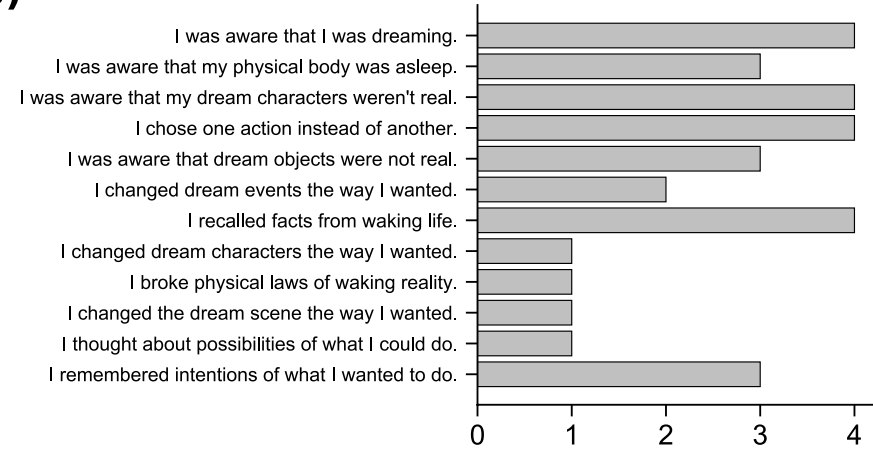

C)

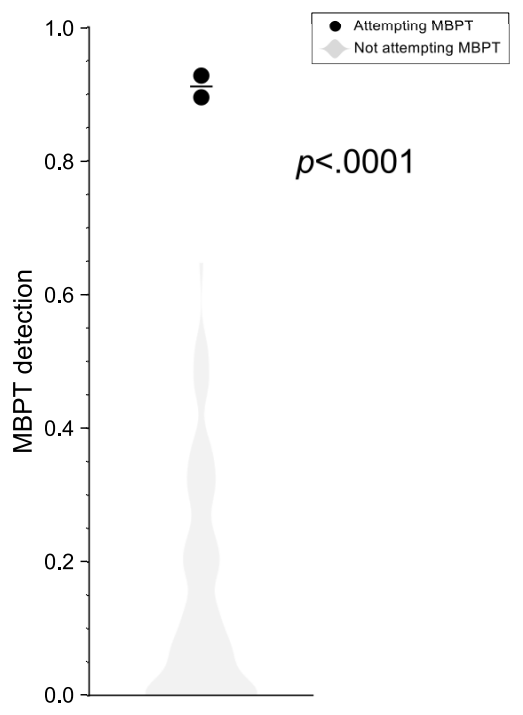

Figure 3. Results from sub-003 lucid dream MBPT task completion. A) DLQ scores. Likert responses upon awakening qualitatively indicate clear lucidity during the dream (LuCiD scores in Figure S3). B) Dream report. Excerpts from the dream report indicate lucidity and task completion (full dream report in Figure S4). C) $\mathrm{BCl}$ detection during sleep. MBPT performed during dreaming is easily detectable with a wake-trained $\mathrm{BCl}$. See Analysis section for description of the MBPT detection measure. Horizontal bar is the mean MBPT detection score of the two segments following LRLR signaling (see footnote for video excerpt of task completion during sleep).

Participant sub-004 performed the training phase while sitting upright at a desk, directly in front of the laptop. The training phase in total took approximately 90 minutes (with breaks) because there was less success in training the $\mathrm{BCl}$ (null distribution 234 confidence interval $=[0, .76]$; Figure 4C), possibly due to a weaker headset connection. 235 Participant sub-004 went to sleep according to their regular nightly schedule, but woke 236 up approximately five hours after sleep, performed the training phase, and then returned 237 to sleep. Participant sub-004 reported becoming lucid and completing the MBPT once 238 following LRLR signaling during a lucid dream (Figure 4A), and DLQ scores suggest the 239 dream was indeed lucid (Figure 4B). BCl detection analysis shows mild support for 240 objective task detection ( $p=.13$; Figure $4 C$ ) with a lucid dream MBPT detection score of 2410.55 following LRLR eye signaling (onset of dream task). 


\section{sub-004}

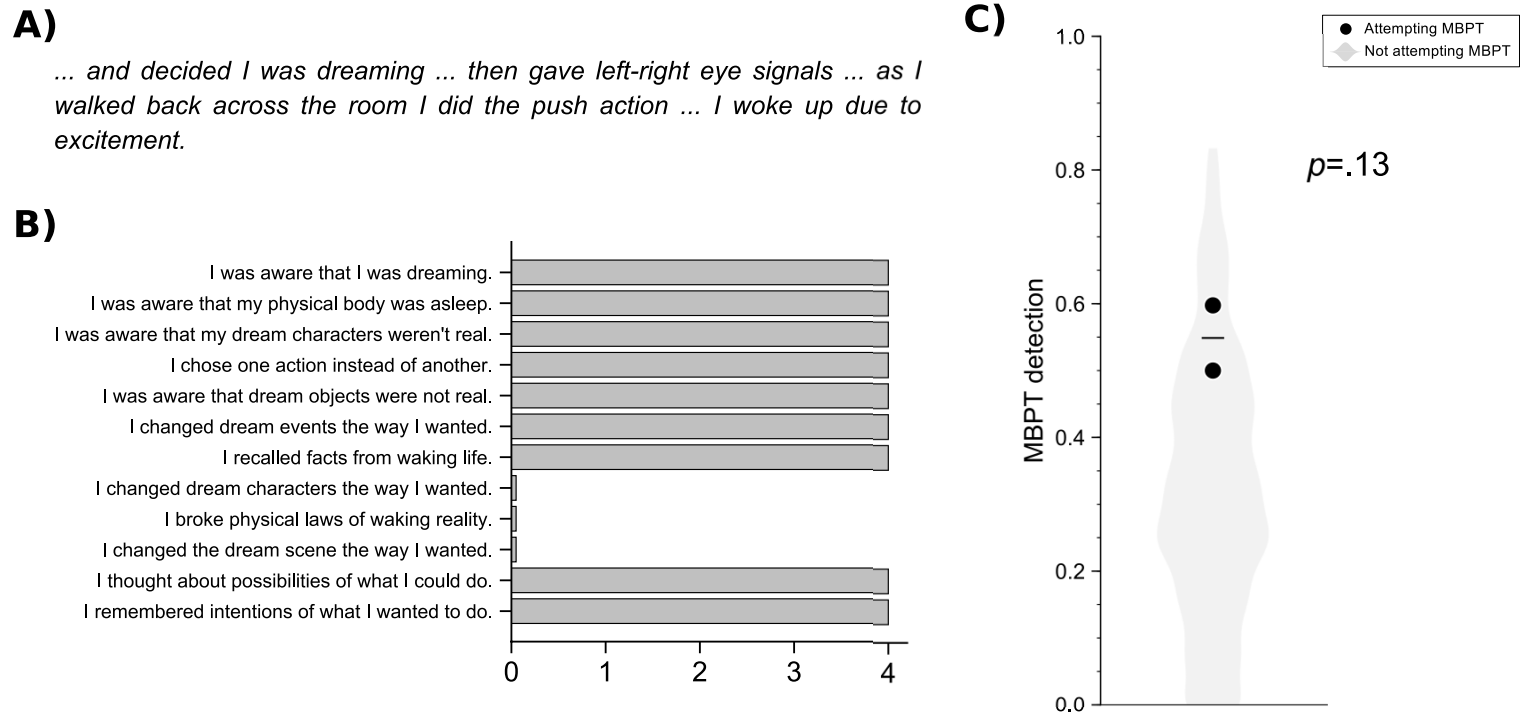

Figure 4. Results from sub-004 lucid dream task completion. A) DLQ scores. Likert responses upon awakening qualitatively indicate clear lucidity during the dream (LuCiD scores in Figure S3). B) Dream report. Excerpts from the dream report indicate lucidity and task completion (full dream report in Figure S4). C) BCl detection during sleep. MBPT performed during dreaming is mildly detectable with a wake-trained $\mathrm{BCl}$. See Analysis section for description of the MBPT detection measure. Horizontal bar is the mean MBPT detection score of the two segments following LRLR signaling.

\section{Discussion}

The present pilot data show are preliminary evidence suggesting that $\mathrm{BCls}$ developed for waking control with mental imagery (Chaudhary et al., 2016; Lebedev \& Nicolelis, 2006; Wolpaw et al., 2002) can be similarly used from within a lucid dream.

248 Three participants with high lucid dream induction success rates reported performing a specific mental task during a lucid dream in an attempt to activate a consumer-grade $\mathrm{BCl}$ that was trained on the same mental task during waking. Of the two participants whose data was successfully recorded through the sleep session, one showed clear evidence of the $\mathrm{BCl}$ detecting the task and the other showed mild evidence. The participant who showed less evidence also had lower signal quality during $\mathrm{BCl}$ training and testing. Importantly, this study includes many caveats and limitations and necessitates further investigations that involve more sophisticated $\mathrm{BCl}$ technology and analyses before drawing firm conclusions.

The $\mathrm{BCl}$ used in the current study was trained to distinguish only between the presence and absence of a single mental task (i.e., the MBPT), which offers no advancement beyond the already common LRLR eye signaling frequently used in the literature (Baird et al., 2019). This restriction was applied for pilot purposes, but if a single 
mental task can be detected, then increasing the range of detectable tasks should also be plausible. Such an increase in message options would provide a more efficient mode of communication from sleep to waking. With further verification of lucid dreaming $\mathrm{BCl}$ control, the improvement in communication abilities from a sleeping participant to a waking researcher is a promising application that could benefit both research and clinical fields (Appel et al., 2018).

For research, an increase in communication abilities would allow for live dream reporting rather than relying on waking dream recall (Windt, 2013), which has its own limitations (Rosen, 2013; Solomonova, Fox, \& Nielsen, 2014). Additionally, lucid dreaming offers a rare state of consciousness with its own neurophysiological signature (Dresler et al., 2012; Voss, Holzmann, Tuin, \& Hobson, 2009), and probing cognition during this state is an intriguing avenue of consciousness research (Appel et al., 2018; Baird et al., 2019; Windt \& Noreika, 2011). Probing the lucid dreaming brain during sleep is a promising experimental approach (Appel, 2013; Appel \& Pipa, 2017; Konkoly \& Paller, 2019), and increasing response options from the dream with imagery and motor actions has the potential to increase experiment complexity. Furthermore, lucid dreaming has been consistently linked with creativity (Blagrove \& Hartnell, 2000; Stumbrys \& Daniels, 2010), but a recent suggestion is that creative solutions that occur during dreaming are less- or in-accessible upon awakening (Stumbrys \& Daunytè, 2018). Being able to control computer signals from a dream - either to send messages or to directly control other tools - opens avenues for extracting that creativity.

$\mathrm{BCl}$ research is largely motivated by developing communication tools for those who are currently restricted by clinical conditions (Chatelle et al., 2012; Chaudhary et al., 2016; Luauté, Morlet, \& Mattout, 2015; Wolpaw et al., 2002) such as motor disorders (Bauer, Gerstenbrand, \& Rumpl, 1979) or disorders of consciousness (Bernat, 2006; Owen, 2008). In extreme cases, it is unclear if such patients are conscious. Thus, a crucial line of research is to develop response options for immobilized patients (Chatelle et al., 2012; Monti et al., 2010; Owen et al., 2006; Owen, Schiff, \& Laureys, 2016). While patient populations are currently used in the development of $\mathrm{BCl}$ communication tools, these experiments are often unable to distinguish whether the patient is not conscious or just unable to control the $\mathrm{BCl}$ (Overgaard \& Overgaard, 2011; Peterson, Cruse, Naci, Weijer, \& Owen, 2015). Most lucid dreams occur in REM sleep (LaBerge, 1988; LaBerge et al., 1986), which includes muscle atonia that prevents most overt motor actions (Chase \& Morales, 1990). This physiological similarity suggests that lucid dreaming participants might serve as a viable model for developing methods to communicate with clinically immobilized patients.

Performing an action during a dream can itself be considered a form of mental imagery, since the entire experience is internally generated. Interestingly, it is also 
300 to the dream state in that there are two levels of internal representation involved; the 301 dream character - already an internal representation - is able to generate another internal 302 representation using mental imagery. In the current study, participants were instructed to 303 use mental imagery for the MBPT during waking and then simply repeat the task during 304 their lucid dream. Explicit instructions as to whether they should act out or imagine the 305 MBPT during their dream wasn't specified, and which of these two alternatives they chose 306 was unclear from initial dream reports (see SI for full dream reports). The prediction here 307 that a $\mathrm{BCl}$ could be controlled during a lucid dream was based on research into the neural 308 overlap between action, imagery, and lucid dream actions (Dresler et al., 2011), but future 309 work might distinguish between the neural representations of lucid dream actions and 310 lucid dream imagery.

311 To my knowledge, the current pilot exploration is the first to demonstrate that a $312 \mathrm{BCl}$ trained on waking imagery can be controlled with similar intentions during a lucid 313 dream. The present use of consumer-grade $\mathrm{BCl}$ equipment and only a few participants 314 prevent strong inferences but indicate that further research should be conducted to 315 evaluate whether BCls will serve as an efficient mode of communication from sleep. 


\section{REFERENCES}

318 Appel, K. (2013). Communication with a sleeping person (Master's). University of

Appel, K. (2019, July). Communication from lucid REM sleep using the swEYEpe method. Talk presented at the Metacognition night and day: lucid dreaming, mind wandering and mindfulness, Nijmegen, The Netherlands.

Appel, K., \& Pipa, G. (2017). Auditory evoked potentials in lucid dreams: A dissertation summary. International Journal of Dream Research, 98-100. https://doi.org/10.11588/ijodr.2017.1.37192

Appel, K., Pipa, G., \& Dresler, M. (2018). Investigating consciousness in the sleep laboratory - an interdisciplinary perspective on lucid dreaming. Interdisciplinary Science Reviews, 43(2), 192-207. https://doi.org/10.1080/03080188.2017.1380468

Badcock, N. A., Mousikou, P., Mahajan, Y., de Lissa, P., Thie, J., \& McArthur, G. (2013). Validation of the Emotiv EPOC EEG gaming system for measuring research quality auditory ERPs. PeerJ, 1, e38. https://doi.org/10.7717/peerj.38

Baird, B., Mota-Rolim, S. A., \& Dresler, M. (2019). The cognitive neuroscience of lucid dreaming. Neuroscience \& Biobehavioral Reviews, 100, 305-323. https://doi.org/10.1016/j.neubiorev.2019.03.008

Bauer, G., Gerstenbrand, F., \& Rumpl, E. (1979). Varieties of the locked-in syndrome. Journal of Neurology, 221(2), 77-91. https://doi.org/10.1007/BF00313105

Bernat, J. L. (2006). Chronic disorders of consciousness. The Lancet, 367(9517), 11811192. https://doi.org/10.1016/S0140-6736(06)68508-5

Blagrove, M., \& Hartnell, S. J. (2000). Lucid dreaming: Associations with internal locus of control, need for cognition and creativity. Personality and Individual Differences, 28(1), 41-47. https://doi.org/10.1016/S0191-8869(99)00078-1

Bobrov, P., Frolov, A., Cantor, C., Fedulova, I., Bakhnyan, M., \& Zhavoronkov, A. (2011). Brain-Computer Interface Based on Generation of Visual Images. PLOS ONE, 6(6), e20674. https://doi.org/10.1371/journal.pone.0020674

Chase, M. H., \& Morales, F. R. (1990). The atonia and myoclonia of active (REM) sleep. Annual Review of Psychology, 41, 557-584. https://doi.org/10.1146/annurev.ps.41.020190.003013

Chatelle, C., Chennu, S., Noirhomme, Q., Cruse, D., Owen, A. M., \& Laureys, S. (2012). Brain-computer interfacing in disorders of consciousness. Brain Injury, 26(12), 1510-1522. https://doi.org/10.3109/02699052.2012.698362

Chaudhary, U., Birbaumer, N., \& Ramos-Murguialday, A. (2016). Brain-computer interfaces for communication and rehabilitation. Nature Reviews Neurology, 12(9), 513-525. https://doi.org/10.1038/nrneurol.2016.113

De Vos, M., Kroesen, M., Emkes, R., \& Debener, S. (2014). P300 speller BCI with a mobile EEG system: Comparison to a traditional amplifier. Journal of Neural Engineering, 11(3), 036008. https://doi.org/10.1088/1741-2560/11/3/036008 Debener, S., Minow, F., Emkes, R., Gandras, K., \& Vos, M. de. (2012). How about taking a low-cost, small, and wireless EEG for a walk? Psychophysiology, 49(11), 1617-1621. https://doi.org/10.1111/j.1469-8986.2012.01471.x 
361

362

363

364

365

366

367

368

369

370

371

372

373

374

375

376

377

378

379

380

381

382

383

384

385

386

387

388

389

390

391

392

393

394

395

396

397

398

399

400

401

402

403

404
Dodet, P., Chavez, M., Leu-Semenescu, S., Golmard, J.-L., \& Arnulf, I. (2015). Lucid Dreaming in Narcolepsy. Sleep, 38(3), 487-497. https://doi.org/10.5665/sleep.4516

Dresler, M., Eibl, L., Fischer, C. F., Wehrle, R., Spoormaker, V. I., Steiger, A., ... Pawlowski, M. (2014). Volitional components of consciousness vary across wakefulness, dreaming and lucid dreaming. Frontiers in Psychology, 4. https://doi.org/10.3389/fpsyg.2013.00987

Dresler, M., Koch, S. P., Wehrle, R., Spoormaker, V. I., Holsboer, F., Steiger, A., ... Czisch, M. (2011). Dreamed Movement Elicits Activation in the Sensorimotor Cortex. Current Biology, 21(21), 1833-1837. https://doi.org/10.1016/j.cub.2011.09.029

Dresler, M., Wehrle, R., Spoormaker, V. I., Koch, S. P., Holsboer, F., Steiger, A., ... Czisch, M. (2012). Neural Correlates of Dream Lucidity Obtained from Contrasting Lucid versus Non-Lucid REM Sleep: A Combined EEG/fMRI Case Study. Sleep, 35(7), 1017-1020. https://doi.org/10.5665/sleep.1974

Erlacher, D., Schädlich, M., Stumbrys, T., \& Schredl, M. (2014). Time for actions in lucid dreams: Effects of task modality, length, and complexity. Frontiers in Psychology, 4. https://doi.org/10.3389/fpsyg.2013.01013

Erlacher, D., \& Schredl, M. (2008). Do REM (lucid) dreamed and executed actions share the same neural substrate? International Journal of Dream Research, 714. https://doi.org/10.11588/ijodr.2008.1.20

Fenwick, P., Schatzman, M., Worsley, A., Adams, J., Stone, S., \& Baker, A. (1984). Lucid dreaming: Correspondence between dreamed and actual events in one subject during rem sleep. Biological Psychology, 18(4), 243-252. https://doi.org/10.1016/0301-0511(84)90056-5

Hearne, K. M. (1978). Lucid dreams: An electro-physiological and psychological study (Ph.D.). University of Liverpool, England.

Hunter, J. D. (2007). Matplotlib: A 2D Graphics Environment. Computing in Science \& Engineering, 9(3), 90-95. https://doi.org/10.1109/MCSE.2007.55

Konkoly, K., \& Paller, K. A. (2019, June). Two-way communication between dreamers and experimenters. Poster presented at the 36th Annual Conference of the International Association for the Study of Dreams, Kerkrade, The Netherlands.

LaBerge, S. (1980). Lucid Dreaming as a Learnable Skill: A Case Study. Perceptual and Motor Skills, 51(3_suppl2), 1039-1042. https://doi.org/10.2466/pms.1980.51.3f.1039

LaBerge, S. (1988). The Psychophysiology of Lucid Dreaming. In J. Gackenbach \& S. LaBerge (Eds.), Conscious Mind, Sleeping Brain: Perspectives on Lucid Dreaming (pp. 135-153). https://doi.org/10.1007/978-1-4757-0423-5_7

LaBerge, S., Baird, B., \& Zimbardo, P. G. (2018). Smooth tracking of visual targets distinguishes lucid REM sleep dreaming and waking perception from imagination. Nature Communications, 9(1), 3298. https://doi.org/10.1038/s41467-018-05547-0

LaBerge, S., Levitan, L., \& Dement, W. C. (1986). Lucid Dreaming: Physiological Correlates of Consciousness during REM Sleep. The Journal of Mind and Behavior, 7(2/3), 251-258. 
405

406

407

408

409

410

411

412

413

414

415

416

417

418

419

420

421

422

423

424

425

426

427

428

429

430

431

432

433

434

435

436

437

438

439

440

441

442

443

444

445

446
LaBerge, S., Nagel, L. E., Dement, W. C., \& Zarcone, V. P. (1981). Lucid Dreaming Verified by Volitional Communication during Rem Sleep. Perceptual and Motor Skills, 52(3), 727-732. https://doi.org/10.2466/pms.1981.52.3.727

Lebedev, M. A., \& Nicolelis, M. A. L. (2006). Brain-machine interfaces: Past, present and future. Trends in Neurosciences, 29(9), 536-546. https://doi.org/10.1016/j.tins.2006.07.004

Luauté, J., Morlet, D., \& Mattout, J. (2015). BCl in patients with disorders of consciousness: Clinical perspectives. Annals of Physical and Rehabilitation Medicine, 58(1), 29-34. https://doi.org/10.1016/j.rehab.2014.09.015

McKinney, W. (2010). Data structures for statistical computing in python. Proceedings of the 9th Python in Science Conferene, 445, 51-56. Retrieved from http://conference.scipy.org/proceedings/scipy2010/mckinney.html

Monti, M. M., Vanhaudenhuyse, A., Coleman, M. R., Boly, M., Pickard, J. D., Tshibanda, L., ... Laureys, S. (2010). Willful Modulation of Brain Activity in Disorders of Consciousness. New England Journal of Medicine, 362(7), 579589. https://doi.org/10.1056/NEJMoa0905370

Oliphant, T. E. (2006). A guide to NumPy (Vol. 1). USA: Trelgol Publishing.

Oudiette, D., Dodet, P., Ledard, N., Artru, E., Rachidi, I., Similowski, T., \& Arnulf, I. (2018). REM sleep respiratory behaviours match mental content in narcoleptic lucid dreamers. Scientific Reports, 8(1), 2636. https://doi.org/10.1038/s41598018-21067-9

Overgaard, M., \& Overgaard, R. (2011). Measurements of consciousness in the vegetative state. The Lancet, 378(9809), 2052-2054. https://doi.org/10.1016/S0140-6736(11)61591-2

Owen, A. M. (2008). Disorders of Consciousness. Annals of the New York Academy of Sciences, 1124(1), 225-238. https://doi.org/10.1196/annals.1440.013

Owen, A. M., Coleman, M. R., Boly, M., Davis, M. H., Laureys, S., \& Pickard, J. D. (2006). Detecting Awareness in the Vegetative State. Science, 313(5792), 14021402. https://doi.org/10.1126/science.1130197

Owen, A. M., Schiff, N. D., \& Laureys, S. (2016). The Assessment of Conscious Awareness in the Vegetative State. In S. Laureys, O. Gosseries, \& G. Tononi (Eds.), The Neurology of Conciousness (Second Edition) (pp. 155-166). https://doi.org/10.1016/B978-0-12-800948-2.00010-8

Perez, F., \& Granger, B. E. (2007). IPython: A System for Interactive Scientific Computing. Computing in Science \& Engineering, 9(3), 21-29. https://doi.org/10.1109/MCSE.2007.53

Peterson, A., Cruse, D., Naci, L., Weijer, C., \& Owen, A. M. (2015). Risk, diagnostic error, and the clinical science of consciousness. Neurolmage: Clinical, 7, 588597. https://doi.org/10.1016/j.nicl.2015.02.008

Price, R. F., \& Cohen, D. B. (1988). Lucid Dream Induction. In J. Gackenbach \& S. LaBerge (Eds.), Conscious Mind, Sleeping Brain: Perspectives on Lucid Dreaming (pp. 105-134). https://doi.org/10.1007/978-1-4757-0423-5_6 
447 Rak, M., Beitinger, P., Steiger, A., Schredl, M., \& Dresler, M. (2015). Increased Lucid

Dreaming Frequency in Narcolepsy. Sleep, 38(5), 787-792. https://doi.org/10.5665/sleep.4676

Rosen, M. G. (2013). What I make up when I wake up: Anti-experience views and narrative fabrication of dreams. Frontiers in Psychology, 4. https://doi.org/10.3389/fpsyg.2013.00514

Schatzman, M., Worsley, A., \& Fenwick, P. (1988). Correspondence during Lucid Dreams between Dreamed and Actual Events. In J. Gackenbach \& S. LaBerge (Eds.), Conscious Mind, Sleeping Brain: Perspectives on Lucid Dreaming (pp. 155-179). https://doi.org/10.1007/978-1-4757-0423-5_8

Solomonova, E., Fox, K. C. R., \& Nielsen, T. (2014). Methodological considerations for the neurophenomenology of dreaming: Commentary on Windt's "Reporting dream experience." Frontiers in Human Neuroscience, 8. https://doi.org/10.3389/fnhum.2014.00317

Stumbrys, T., \& Daniels, M. (2010). An exploratory study of creative problem solving in lucid dreams: Preliminary findings and methodological considerations. International Journal of Dream Research, 121-129. https://doi.org/10.11588/ijodr.2010.2.6167

Stumbrys, T., \& Daunytè, V. (2018). Visiting the land of dream muses: The relationship between lucid dreaming and creativity. International Journal of Dream Research, 207-212. https://doi.org/10.11588/ijodr.2018.2.48667

Stumbrys, T., Erlacher, D., Johnson, M., \& Schredl, M. (2014). The Phenomenology of Lucid Dreaming: An Online Survey. The American Journal of Psychology, 127(2), 191-204. Retrieved from JSTOR.

Stumbrys, T., Erlacher, D., Schädlich, M., \& Schredl, M. (2012). Induction of lucid dreams: A systematic review of evidence. Consciousness and Cognition, 21(3), 1456-1475. https://doi.org/10.1016/j.concog.2012.07.003

Stumbrys, T., Erlacher, D., \& Schredl, M. (2013). Testing the involvement of the prefrontal cortex in lucid dreaming: A tDCS study. Consciousness and Cognition, 22(4), 1214-1222. https://doi.org/10.1016/j.concog.2013.08.005

Taylor, G. S., \& Schmidt, C. (2012). Empirical Evaluation of the Emotiv EPOC BCI Headset for the Detection of Mental Actions. Proceedings of the Human Factors and Ergonomics Society Annual Meeting, 56(1), 193-197. https://doi.org/10.1177/1071181312561017

Voss, U., Holzmann, R., Tuin, I., \& Hobson, A. J. (2009). Lucid Dreaming: A State of Consciousness with Features of Both Waking and Non-Lucid Dreaming. Sleep, 32(9), 1191-1200. https://doi.org/10.1093/sleep/32.9.1191

Voss, U., Schermelleh-Engel, K., Windt, J., Frenzel, C., \& Hobson, A. (2013). Measuring consciousness in dreams: The lucidity and consciousness in dreams scale. Consciousness and Cognition, 22(1), 8-21. https://doi.org/10.1016/j.concog.2012.11.001

Windt, J. M. (2013). Reporting dream experience: Why (not) to be skeptical about dream reports. Frontiers in Human Neuroscience, 7. https://doi.org/10.3389/fnhum.2013.00708 
491 Windt, J. M., \& Noreika, V. (2011). How to integrate dreaming into a general theory of 492 consciousness - A critical review of existing positions and suggestions for future

493

494 495 496 research. Consciousness and Cognition, 20(4), 1091-1107. https://doi.org/10.1016/j.concog.2010.09.010

Wolpaw, J. R., Birbaumer, N., McFarland, D. J., Pfurtscheller, G., \& Vaughan, T. M. (2002). Brain-computer interfaces for communication and control. Clinical Neurophysiology, 113(6), 767-791. https://doi.org/10.1016/S13882457(02)00057-3

Zadra, A. (2016, June). Memory and imagination: The stuff of dreams? Talk presented at the 33rd Annual Conference of the International Association for the Study of Dreams, Kerkrade, The Netherlands.

Zich, C., De Vos, M., Kranczioch, C., \& Debener, S. (2015). Wireless EEG with individualized channel layout enables efficient motor imagery training. Clinical Neurophysiology, 126(4), 698-710. https://doi.org/10.1016/j.clinph.2014.07.007 